\title{
Article \\ Electron Diffraction Study of the Space Group Variation in the Al-Mn-Pt T-Phase
}

\author{
Rimon Tamari ${ }^{1}$, Benjamin Grushko ${ }^{2,3}$ and Louisa Meshi ${ }^{1, *(D)}$ \\ 1 Department of Materials Engineering, Ben Gurion University of the Negev, Beer Sheva 8410501, Israel; \\ rimonta@post.bgu.ac.il \\ 2 MaTecK GmbH, 52428 Julich, Germany; B.Grushko@fz-juelich.de \\ 3 Peter-Grünberg-Institut, Forschungszentrum Jülich, 52425 Julich, Germany \\ * Correspondence: louisa@bgu.ac.il; Tel.: +972-8-647-2576
}

Citation: Tamari, R.; Grushko, B.; Meshi, L. Electron Diffraction Study of the Space Group Variation in the Al-Mn-Pt T-Phase. Symmetry 2022, 14, 38. https://doi.org/10.3390/ sym14010038

Academic Editors: Partha Pratim Das, Arturo Ponce-Pedraza, Enrico

Mugnaioli and Stavros Nicolopoulos

Received: 15 November 2021

Accepted: 27 December 2021

Published: 29 December 2021

Publisher's Note: MDPI stays neutral with regard to jurisdictional claims in published maps and institutional affiliations.

Copyright: (c) 2021 by the authors. Licensee MDPI, Basel, Switzerland. This article is an open access article distributed under the terms and conditions of the Creative Commons Attribution (CC BY) license (https:// creativecommons.org/licenses/by/ $4.0 /)$.

\begin{abstract}
Binary high temperature " $\mathrm{Al}_{3} \mathrm{Mn}$ " (T-phase) and its extensions in ternary systems were the subjects of numerous crystallographic investigations. The results were ambiguous regarding the existence or lack of the center of symmetry: both Pna2 $2_{1}$ and Pnam space groups were reported. Our research on the Al-Mn-Pt T-phase allowed concluding that inside a continuous homogeneity region, the structure of the Al-rich T-phase (e.g., $\mathrm{Al}_{78} \mathrm{Mn}_{17.5} \mathrm{Pt}_{4.5}$ ) belongs to the non-centrosymmetric $\mathrm{Pna}_{1}$ space group, while the structure of the Al-poor T-phase (such as $\mathrm{Al}_{71.3} \mathrm{Mn}_{25.1} \mathrm{Pt}_{3.6}$ ) is centrosymmetric, i.e., Pnam. Following metallurgical and crystallographic considerations, the change in the symmetry was explained.
\end{abstract}

Keywords: $\mathrm{Al}_{3} \mathrm{Mn}$; space group; Convergent Beam Electron Diffraction (CBED); center of symmetry; aluminide

\section{Introduction}

The Al-Mn so-called T-phase, also known as high-temperature " $\mathrm{Al}_{3} \mathrm{Mn}$ ", is formed in a compositional region between $\sim 25$ and 29 at. \% $\mathrm{Mn}$ and a temperature range of $895-1002{ }^{\circ} \mathrm{C}$ (see [1] and references therein). The compositional region of the T-phase widely extends in numerous ternary $\mathrm{Al}-\mathrm{MnM}$ alloy systems, where $\mathrm{M}$ is transition metal (see [2] and references therein), which results in a significant decrease in its stability temperature. Furthermore, it was argued that the binary stoichiometry of the T-phase is $\mathrm{Al}_{124} \mathrm{Mn}_{32}$, which is outside of its homogeneity region [2]. Reports on the atomic structure of the T-phase are puzzling. The history of the structure determination of the T-phase is a perfect example of a dispute about the accuracy of the estimation of the space group and corresponding atomic models. In 1938, the geometry of the T-phase's unit cell was reported by Hoffmann as orthorhombic [3]. In 1961, the centrosymmetric Pnam space group was chosen to describe its symmetry [4], while in 1992, in a series of publications by Li et al. (see [5] and references therein), the non-centrosymmetric Pna2 ${ }_{1}$ was argued. In 1993, its atomic model was proposed again in the framework of the Pnam space group [6], while in 1994, Shi et al. [7] insisted on Pna2 ${ }_{1}$. In 1995, Pavlyuk et al. [8] succeeded in refining T phase's structure in the framework of the Pnam space group but failed when using Pna2 1 , which, according to these authors, would not give significantly better results. The binary Pnam prototype was chosen as a start for the structure refinement of the ternary T-phases [9-13]. Despite the common starting model, their final atomic models varied. The major discrepancies were found in the occupancies and positions of transition metals.

Recently, the extension of the T-phase region in the Al-Mn-Pt alloy system up to $\sim \mathrm{Al}_{78} \mathrm{Mn}_{17.5} \mathrm{Pt}_{4.5}$ and its stability at ternary compositions down to at least $800{ }^{\circ} \mathrm{C}$ were reported in [2]. A further study confirmed its stability also at $700{ }^{\circ} \mathrm{C}$ (to be published). The following lattice parameters were determined for the above-mentioned composition: $a=14.720(4) \AA, b=12.628(2) \AA, c=12.545(3) \AA$ [2]. 
By using electron diffraction methods, a partial atomic model of the $\mathrm{Al}_{78} \mathrm{Mn}_{17.5} \mathrm{Pt}_{4.5}$ T-phase was proposed in the framework of the non-centrosymmetric Pna2 ${ }_{1}$ space group [2]. The current research was undertaken with the purpose of relating the above-mentioned inconsistency in the space group to differences in the composition of the ternary T-phase. It should be mentioned that ternary extensions of the T-phase, discussed in [2], are much wider than the above-mentioned binary T-phase region. We performed a study of the symmetry of the Al-Mn-Pt T-phase varying the annealing temperature $\left(700\right.$ vs. $\left.800{ }^{\circ} \mathrm{C}\right)$ as well as its composition: $\mathrm{Al}_{78} \mathrm{Mn}_{17.5} \mathrm{Pt}_{4.5}$ vs. $\mathrm{Al}_{71.3} \mathrm{Mn}_{25.1} \mathrm{Pt}_{3.6}$. It was found that, regardless of the annealing temperature, the $\mathrm{Al}_{78} \mathrm{Mn}_{17.5} \mathrm{Pt}_{4.5} \mathrm{~T}$-phase is non-centrosymmetric, while the unit cell of the $\mathrm{Al}_{71.3} \mathrm{Mn}_{25.1} \mathrm{Pt}_{3.6} \mathrm{~T}$-phase can be described by the centrosymmetric Pnam space group. Furthermore, a direct structural relationship between the two options (centrosymmetric vs. non-centrosymmetric) is proposed.

\section{Materials and Methods}

Two Al-Mn-Pt compositions (see Table 1) were selected from the opposite Al limits of the compositional region of the T-phase. The alloys were produced by levitation induction melting in a water-cooled copper crucible under a pure Ar atmosphere. The purity of $\mathrm{Al}$ was $99.999 \%$, Mn 99.99\%, and Pt 99.9\%. For homogeneity, the samples were annealed under a vacuum of $9 \times 10^{-7} \mathrm{mBar}$.

Table 1. Al-Mn-Pt alloys studied in this research.

\begin{tabular}{ll}
\hline Nominal Composition & Heat Treatment Details \\
\hline $\mathrm{Al}_{78} \mathrm{Mn}_{17.5} \mathrm{Pt}_{4.5}$ & $800{ }^{\circ} \mathrm{C}$ for $622 \mathrm{~h}$ \\
$\mathrm{Al}_{78} \mathrm{Mn}_{17.5} \mathrm{Pt}_{4.5}$ & $700{ }^{\circ} \mathrm{C}$ for $984 \mathrm{~h}$ \\
$\mathrm{Al}_{71.3} \mathrm{Mn}_{25.1} \mathrm{Pt}_{3.6}$ & $800{ }^{\circ} \mathrm{C}$ for $649 \mathrm{~h}$ \\
\hline
\end{tabular}

The alloys were ground into powder using an agate mortar and pestle, dispersed in isopropanol, and stirred in the ultrasonic bath. Each suspension was drop cast on a carbon-coated $\mathrm{Cu}$ grid. The samples were studied using the JEOL JEM-2100 Transmission Electron Microscope (TEM) operating at $200 \mathrm{kV}$. A convergence angle of about $2 \times 10^{-2} \mathrm{rad}$ was used to acquire Convergent Beam Electron Diffraction (CBED) patterns. A condenser aperture of $20 \mu \mathrm{m}$ in diameter was applied to eliminate the overlapping of the CBED disks.

The Pnam and Pna2 ${ }_{1}$ space groups, discussed here, exhibit the same extinction conditions: $\mathrm{k}+\mathrm{l}=2 \mathrm{n}$ for the $\{0 \mathrm{kl}\}$ type reflections; $\mathrm{h}=2 \mathrm{n}$ for the $\{\mathrm{h} 0 \mathrm{l}\}$ and $\{\mathrm{h} 00\}$ type reflections; $\mathrm{k}=2 \mathrm{n}$ and $\mathrm{l}=2 \mathrm{n}$ for the $\{0 \mathrm{k} 0\}$ and $\{001\}$ type reflections [14]. These space groups could not be distinguished by powder X-ray diffraction and conventional selected area electron diffraction (SAED). On the other hand, the CBED method has the power to perform this, differentiating between not only the extinction conditions but also the point groups. It is based on an evaluation of the highly sensitive to symmetry Zero Order Laue Zone (ZOLZ) and the whole patterns (WP), containing the Higher-Order Laue Zones (HOLZ) in addition to ZOLZ. These patterns can be 3D or 2D, depending on the information seen or not seen in the CBED disks. In some cases, even the 2D information found in the CBED patterns is sufficient for an estimation of the correct symmetry. The methodology, along with the definition of the types of patterns used, can be found in [15].

The Pnam and Pna2 1 space groups belong to the mmm and $m m 2$ point groups, respectively, and as stated, exhibit different symmetries of the CBED patterns taken along the high symmetry axes [15]. During the capturing of the whole patterns, due to the above-mentioned large unit cell parameters of the T-phase, it was impossible to avoid the overlapping of the CBED disks. Thus, microdiffraction patterns with almost parallel beams were taken in the nano-beam mode. For convenience, Table 2 is reproduced from [15], summarizing the symmetry of the CBED patterns expected at the high symmetry axes for the $m m m$ and $m m 2$ point groups. 
Table 2. Two-dimensional and 3D bright field (BF) and whole pattern (WP) symmetries of the $\mathrm{mmm}$ and $m m 2$ point groups. This table was reproduced from [15].

\begin{tabular}{|c|c|c|c|c|c|c|}
\hline & [100] & [10] & [1] & [u0w] & [uv0] & [0vw] \\
\hline \multirow{4}{*}{ mmm } & 2D BF $(2 m m)$ & 2D BF $(2 \mathrm{~mm})$ & 2D BF $(2 \mathrm{~mm})$ & 2D BF $(2 \mathrm{~mm})$ & 2D BF $(2 m m)$ & 2D BF $(2 m m)$ \\
\hline & 2D WP $(2 m m)$ & 2D WP $(2 m m)$ & 2D WP $(2 m m)$ & 2D WP $(2 m m)$ & 2D WP $(2 m m)$ & 2D WP $(2 \mathrm{~mm})$ \\
\hline & $3 \mathrm{D}$ BF $2 \mathrm{~mm}$ & $3 \mathrm{D}$ BF $2 \mathrm{~mm}$ & $3 \mathrm{D}$ BF $2 \mathrm{~mm}$ & $3 \mathrm{D} \mathrm{BF} m$ & 3D BF $m$ & 3D BF $m$ \\
\hline & $3 \mathrm{D} W P 2 \mathrm{~mm}$ & $3 \mathrm{D} W P 2 \mathrm{~mm}$ & $3 \mathrm{D} W P 2 \mathrm{~mm}$ & 3D WP $m$ & 3D WP $m$ & 3D WP $m$ \\
\hline \multirow{4}{*}{$m m 2$} & 2D BF (2mm) & 2D BF (2mm) & 2D BF (2mm) & 2D BF (2mm) & 2D BF (2mm) & $2 \mathrm{D} \mathrm{BF}(2 \mathrm{~mm})$ \\
\hline & 2D WP $(m)$ & 2D WP $(m)$ & 2D WP $(2 m m)$ & 2D WP $(m)$ & 2D WP $(m)$ & 2D WP $(m)$ \\
\hline & $3 \mathrm{D}$ BF $2 \mathrm{~mm}$ & $3 \mathrm{D}$ BF $2 \mathrm{~mm}$ & $3 \mathrm{D}$ BF $2 \mathrm{~mm}$ & $3 \mathrm{D} \mathrm{BF} m$ & $3 \mathrm{D} \mathrm{BF} m$ & $3 \mathrm{D} \mathrm{BF} m$ \\
\hline & 3D WP $m$ & $3 \mathrm{DWP} m$ & $3 \mathrm{D} W P 2 \mathrm{~mm}$ & 3D WP $m$ & 3D WP 1 & 3D WP $m$ \\
\hline
\end{tabular}

\section{Results and Discussion}

Primarily, the effect of the structure relaxation on the symmetry of the unit cell was studied. It was previously reported [2] that an $\mathrm{Al}_{78} \mathrm{Mn}_{17.5} \mathrm{Pt}_{4.5}$ alloy, annealed at $800{ }^{\circ} \mathrm{C}$, contained inside the T-phase's matrix fine precipitation of a second phase, formed during cooling from the annealing temperature. This second phase was identified as isostructural to the so-called R-phase, reported to coexist with the T-phase in the Al-Mn-Pd alloy system, where $\mathrm{Al}_{78.6} \mathrm{Mn}_{15.6} \mathrm{Pd}_{5.7} \mathrm{R}$-phase was found to have the $\mathrm{Cmcm}$ space group and following lattice parameters $a=7.76 \AA, b=23.88 \AA, c=12.43 \AA$ [9,16]. The presence of this phase indicates that the precipitates-free regions of the T-phase, which were used for the structure determination in [2], could be supersaturated. Thus, the structure in these regions could have a different space group than that ascribed to the binary T-phase due to the corresponding internal strain. Therefore, the same alloy was additionally annealed at $700{ }^{\circ} \mathrm{C}$ to complete the precipitation of the R-phase, which would result in a relaxation of the T-phase's matrix. By following this annealing, the resultant material contained equiaxed grains of both phases, exceeding $100 \mathrm{~nm}$ in diameter, which is sufficient for electron diffraction experiments.

The unit cell parameters of the T- and R-phases are closely related $[5,9,12]$ and, hence, many interplanar distances $\left(\mathrm{d}_{\mathrm{hkl}}\right)$ overlap. Therefore, at many orientations, electron diffraction patterns could be indexed in terms of both phases. The most effective way to distinguish between them is either by comparing their strong reflections distribution at the principal axes [16] or by the presence of the reflections with the interplanar distance of $\sim 9.5 \AA$ ( $\{110\}$ of the T-phase), which do not appear in the R-phase interplanar spacing list. Therefore, the CBED analysis was performed using the [110] zone axis patterns of the T-phase. According to Table 2, the difference between the discussed point groups ( $\mathrm{mmm}$ vs. $m m 2$ ) along the [1ํㅣㅇㅣ orientation can be observed using the $2 \mathrm{D} \mathrm{WP}$ patterns. This means that only intensity distribution of the CBED disks is considered while assessing the symmetry, i.e., without taking features as excess and deficiency lines, the ZOLZ fringes, etc., into account. This information is available on ZOLZ CBED patterns displaying only ZOLZ disks. The mmm point group will impose the $2 \mathrm{~mm}$ symmetry on the ZOLZ CBED pattern, while the $m$ symmetry of this pattern will indicate that the $m m 2$ point group is the correct one.

The 2D ZOLZ patterns of the $\mathrm{Al}_{78} \mathrm{Mn}_{17.5} \mathrm{Pt}_{4.5} \mathrm{~T}$-phase annealed at 700 and $800{ }^{\circ} \mathrm{C}$ are shown in Figure 1a,b, respectively. Regardless of the annealing temperature, i.e., in either supersaturated or relaxed state, the symmetry of the T-phase remained the same. In both cases, symmetry was evaluated as $m$, indicating the $m m 2$ point group, and subsequently the Pna2 ${ }_{1}$ space group, as reported in [2].

The change in the symmetry as a function of composition can be seen clearly in Figure 1c. For the $\mathrm{Al}_{71.3} \mathrm{Mn}_{25.1} \mathrm{Pt}_{3.6}$ composition (i.e., with a visibly lower $\mathrm{Al} / \mathrm{Mn}$ ratio), the symmetry of the ZOLZ pattern is $2 \mathrm{~mm}$, indicating the $\mathrm{mmm}$ point group and, subsequently, the Pnam space group, which is in line with reported for the binary $\mathrm{T}-$ " $\mathrm{Al}_{3} \mathrm{Mn}$ " phase [6]. 

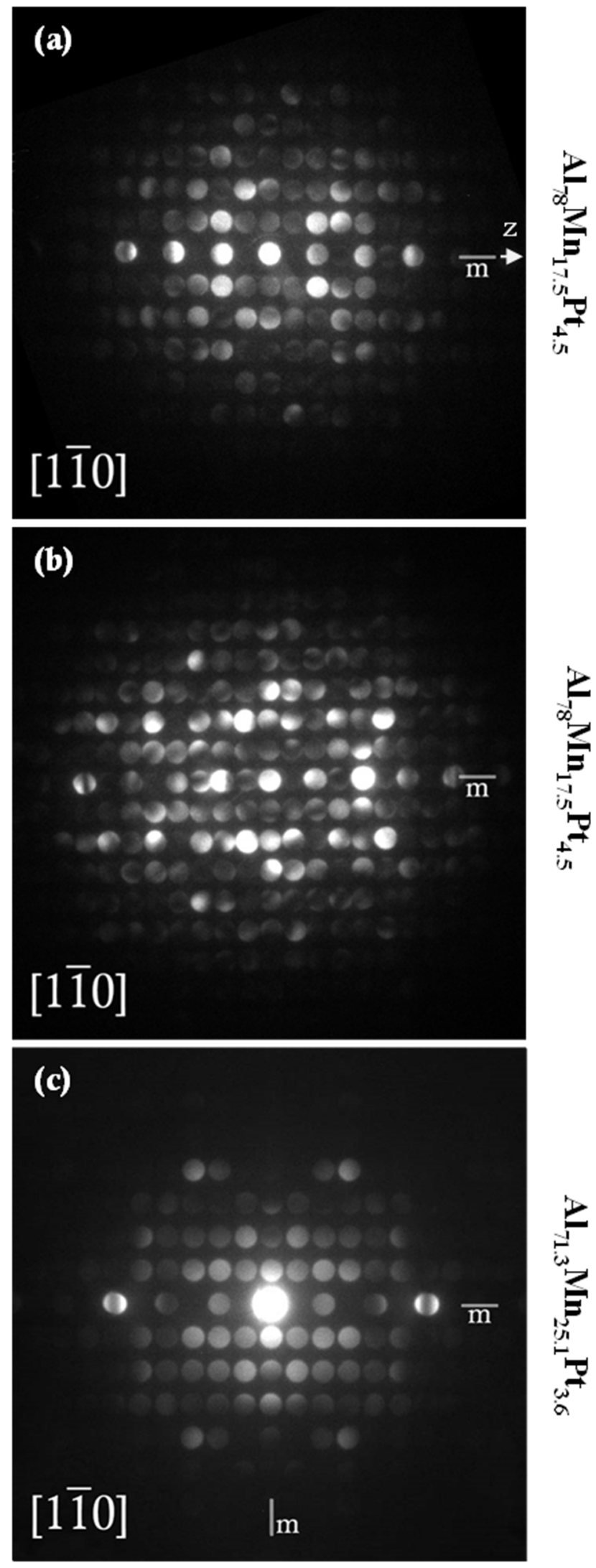

Figure 1. Two-dimensional [1]10] ZOLZ CBED patterns taken from the $\mathrm{Al}_{78} \mathrm{Mn}_{17.5} \mathrm{Pt}_{4.5} \mathrm{~T}$-phase, annealed at $800^{\circ} \mathrm{C}(\mathbf{a})$ and $700{ }^{\circ} \mathrm{C}(\mathbf{b})$ and from the $\mathrm{Al}_{71.3} \mathrm{Mn}_{25.1} \mathrm{Pt}_{3.6} \mathrm{~T}$-phase, annealed at $800{ }^{\circ} \mathrm{C}(\mathbf{c})$. Mirrors are denoted by $m$. [001] direction of the reciprocal space is marked as $z$ on the upper image. All images were aligned to each other. 
The structure of the T-phase with the Pnam space group can be described as a stacking of flat and puckered layers [5]. If these flat layers are not strictly flat, their mirror symmetry is destroyed, resulting in the Pna2 ${ }_{1}$ space group, and a displacement of even \pm 0.005 from $\mathrm{z}=1 / 4$ is sufficient for this. Here, $(\mathrm{x}, \mathrm{y}, 1 / 4)$ is the $4 c$ special Wyckoff position in $a$-cb setting of the Pnma space group, i.e., Pnam, while in the Pna2 1 space group, this position is general, of the $(\mathrm{x}, \mathrm{y}, \mathrm{z})$ type. In a wide compositional region, the replacement between several larger $\mathrm{Al}$ and smaller Mn atoms could easily result in such displacements, which would be more reliably recognized at compositions with an extremal $\mathrm{Al} / \mathrm{Mn}$ ratio. Obviously, this is also the question of the experimental resolution.

On the other hand, due to the change in the space group, some splitting of the corresponding general $8 d$ Wyckoff positions would be inevitable since, in the Pna $2_{1}$ space group, only the 4 a positions exist. Since the maximal translationengleiche subgroup of Pnam is Pna2 1 [17], the transition from one to another will cause the described above splitting of each general $8 d$ Wyckoff position into two general $4 a$ positions: one with the same coordinates, turning to be of the $(x, y, z)$ type, and the other $(0.5-x, 0.5+y, 1-z)$ ). During this transition, each special $4 c$ position (of the $(x, y, 1 / 4)$ type in the Pnam space group) turns into a general position $(\mathrm{x}, \mathrm{y}, \mathrm{z})$ of the $4 a$ type in the Pna2 1 space group [14,17].

Considering the stoichiometry of the T-phase as binary $\mathrm{Al}_{124} \mathrm{Mn}_{32}$, this is plausible to place the $8 \mathrm{Pt}$ atoms in $\mathrm{Al}_{78} \mathrm{Mn}_{17.5} \mathrm{Pt}_{4.5}$ at specific $\mathrm{Mn}$ cites (see [2]), while by decreasing $\mathrm{Al}$ concentration, some $\mathrm{Al}$ sites would be (partially) occupied by $\mathrm{Mn}$, as it is in the case of the current structural models of the T-phase [6,8]. Our results are in favor of a suggestion that the increase in symmetry is rather caused by the replacement of Al by Mn, while the $\mathrm{Mn} / \mathrm{Pt}$ substitution seems to have no influence on the symmetry.

\section{Conclusions}

The current research focuses on the unambiguous determination of the space group of the Al-Mn-Pt T-phase. Our work was motivated by the discussion on the symmetry of the $\mathrm{T}$-phase, reported in binary Al-Mn and various ternary Al-Mn-M (where $\mathrm{M}$ is transition metal) alloy systems, which was held for several decades. By using the CBED method, it was found that the increase in the $\mathrm{Al} / \mathrm{Mn}$ ratio leads to the change from the Pnam to Pna2 ${ }_{1}$ space group. On the other hand, regardless of the annealing temperature, the symmetry of the T-phase remained unchanged. Following metallurgical and crystallographic considerations, an explanation for the symmetry change (centrosymmetric vs. non-centrosymmetric) was proposed.

Author Contributions: Conceptualization, L.M.; methodology, L.M.; software, R.T.; validation, R.T., B.G. and L.M.; formal analysis, R.T.; investigation, R.T.; resources, L.M.; data curation, L.M. and B.G.; writing-original draft preparation, R.T.; writing-review and editing, L.M. and B.G.; visualization, R.T.; supervision, L.M.; funding acquisition, L.M. All authors have read and agreed to the published version of the manuscript.

Funding: This research received no external funding.

Institutional Review Board Statement: Not applicable.

Informed Consent Statement: Not applicable.

Data Availability Statement: Not applicable.

Conflicts of Interest: The authors declare no conflict of interest.

\section{References}

1. McAlister, A.J.; Murray, J.L. The Aluminum-Manganese system. Bull. Alloy. Phase Diagr. 1987, 8, 438-447. [CrossRef]

2. Tamari, R.; Grushko, B.; Meshi, L. Structural study of $\mathrm{Al}_{78} \mathrm{Mn}_{17.5} \mathrm{Pt}_{4.5}$ and (re) constitution of the Al-Mn-Pt system in its vicinity. J. Alloys Compd. 2021, 861, 158328. [CrossRef]

3. Hoffmann, W. Roentgenographic methods in the investigation of Alumium alloys. Aluminium 1938, $20,865-872$.

4. Taylor, M.A. The space group of $\mathrm{MnAl}_{3}$. Acta Crystallogr. 1961, 14, 84. [CrossRef] 
5. Li, X.Z.; Shi, D.; Kuo, K.H. Structure of $\mathrm{Al}_{3} \mathrm{Mn}$, an orthorhombic approximant of the decagonal quasicrystal. Philos. Mag. Part B 1992, 66, 331-340. [CrossRef]

6. Hiraga, K.; Kaneko, M.; Matsuo, Y.; Hashimoto, S. The structure of $\mathrm{Al}_{3} \mathrm{Mn}$ : Close relationship to decagonal quasicrystals. Philos. Mag. Part B 1993, 67, 193-205. [CrossRef]

7. Shi, N.C.; Li, X.Z.; Ma, Z.S.; Kuo, K.H. Crystalline phases related to a decagonal quasicrystal. I. A single-crystal X-ray diffraction study of the orthorhombic $\mathrm{Al}_{3} \mathrm{Mn}$ phase. Acta Crystallogr. B 1994, 50, 22-30. [CrossRef]

8. Pavlyuk, V.V.; Yanson, T.I.; Bodak, O.I.; Černý, R.; Gladyshevskii, R.E.; Yvon, K.; Stepien-Damm, J. Structure refinement of orthorhombic $\mathrm{MnAl}_{3}$. Acta Crystallogr. C 1995, 51, 792-794. [CrossRef]

9. Audier, M.; Durand-Charre, M.; de Boissieu, M. AlPdMn phase diagram in the region of quasicrystalline phases. Philos. Mag. Part B 1993, 68, 607-618. [CrossRef]

10. Matsuo, Y.; Yamamoto, K.; Iko, Y. Structure of a new orthorhombic crystalline phase in the Al-Cr-Pd alloy system. Philos. Mag. Lett. 1997, 75, 137-142. [CrossRef]

11. Matsuo, Y.; Kaneko, M.; Yamanoi, T.; Kaji, N.; Sugiyama, K.; Hiraga, K. The structure of an $\mathrm{Al}_{3} \mathrm{Mn}-\mathrm{type} \mathrm{Al}_{3}(\mathrm{Mn}, \mathrm{Pd}) \mathrm{crystal}$ studied by single-crystal X-ray diffraction analysis. Philos. Mag. Lett. 1997, 76, 357-362. [CrossRef]

12. Klein, H.; Boudard, M.; Audier, M.; de Boissieu, M.; Vincent, H.; Beraha, L.; Duneau, M. The T-Al 3 (Mn, Pd) quasicrystalline approximant: Chemical order and phason defects. Philos. Mag. Lett. 1997, 75, 197-208. [CrossRef]

13. Furihata, J.I.; Okabe, T. Crystalline approximants of decagonal quasicrystals in the Al-Cu-Cr system. Microscopy 1999, 48, 761-766. [CrossRef]

14. Hahn, T. (Ed.) International Tables for Crystallography. Volume A: Space-Group Symmetry; Springer: Berlin, Germany, $2005 ;$ p. 911.

15. Morniroli, J.P. Atlas of Electron Diffraction Zone Axis Patterns. 2013. Available online: http://www.electron-diffraction.fr/ (accessed on 14 November 2021).

16. Balanetskyy, S.; Meisterernst, G.; Heggen, M.; Feuerbacher, M. Reinvestigation of the Al-Mn-Pd alloy system in the vicinity of the T- and R-phases. Intermetallics 2008, 16, 71-87. [CrossRef]

17. Wondratschek, H.; Muller, U. (Eds.) International Tables of Crystallography, Volume A1: Symmetry Relations Between Space Groups; Kluwer Academic Publishers: Amsterdam, The Netherlands, 2004; p. 731. 